\title{
Can Current Quantum Cognition Studies Give Indication on the Manner in Which Human Cognition Arose ab Initio?
}

\author{
Elio Conte ${ }^{1,2}$ \\ ${ }^{1}$ School of Advanced International Studies on Applied Theoretical and Non Linear Methodologies of Physics, \\ Bari, Italy \\ ${ }^{2}$ Department of Neuroscience and Sense Organs, University Aldo Moro, Bari, Italy \\ Email: elio.conte@fastwebnet.it
}

Received 9 April 2014; revised 6 May 2014; accepted 1 June 2014

Copyright (C) 2014 by author and Scientific Research Publishing Inc.

This work is licensed under the Creative Commons Attribution International License (CC BY).

http://creativecommons.org/licenses/by/4.0/

cc) (i) Open Access

\section{Abstract \\ It is advanced the view that the primary elements of cognition and matter both started ab initio as represented in quantum mechanics.}

\section{Keywords}

\section{Quantum Cognition, Cognitive Sciences, Cognition}

\section{Basic Statements}

Cognition is the mental process of knowing, including aspects such as awareness, perception, reasoning, and judgment. It relates what comes to be known, as through perception, reasoning, or intuition and knowledge.

One of our long-range goals is to understand the neural underpinnings of human emotion and examine how cognitive and emotional brain systems interact in the generation of complex behaviour. Cognitive science is presently an advanced discipline but we are currently distant from fully understanding about the advent of cognitive mechanisms and the relationship between cognition and emotion. May be that the current studies on quantum cognition will contribute in the investigation of such basic problem.

The so called quantum cognition has been developed in the recent years. In 1980-1986 the author of the present communication started with some initial contributions. Khrennikov and the present author gave some other contributions starting with 2003 until today. For a comprehensive review one may look at (Khrennikov, 2010) and (Conte, 2011a). Aerts started in 1991 to give contributions (Aerts, 1991) but also previously evidenced the importance to consider deeply the role of quantum mechanics. Other authors as Bruza and Kitto started in 2007, 
Busemeyer and Pathos in 2006 as well as we are able to mention (Busemeyer \& Bruza, 2012). In 1995-2003 Snyder gave also important contributions (Snyder, 1995). Still a lot of authors gave their support so that we may conclude that quantum cognition has had the time to articulate a constant activity of research in a satisfactory manner.

In these years, we have reached decisive experimental evidence on existing quantum interference during perceptive-cognitive performance in humans. We performed experiments by using ambiguous figures but also the Stroop effect and tests based on cognitive anomalies as example the conjunction fallacy.

Let's take a look at one of the foundations of quantum mechanics in a simple way so as to allow even the reader who does not have high and specialized skills in mathematics to follow our reasoning.

Quantum mechanics runs about the notion of observable. We indicate it by $A$.

In quantum mechanics each dynamical variable of a given material system (e.g. position of a particle, translational momentum, orbital angular momentum, spin, total angular momentum, energy, etc.) is associated with a Hermitean operator that acts on the state of the quantum system and whose eigenvalues correspond to the possible values of the dynamical variable that we estimate in a probabilistic manner as a result of a measurement in our laboratory.

We have here a fundamental relationship which revolves around the thesis of this communication. The observable $A$ may be written in the following manner

$$
A=\sum_{i=1}^{k} a_{i} P_{i}
$$

that is to say $A=a_{1} P_{1}+a_{2} P_{2}+\cdots+a_{k} P_{k}$.

$a_{1}, a_{2}, \cdots, a_{k}$ are the eigenvalues that we mentioned previously and this is to say the possible values of the dynamical variable that we are estimating in a probabilistic manner and as result of a measurement while instead $P_{1}, P_{2}, \cdots, P_{k}$ are technically called eigenprojectors and actually we may consider that, according with von Neumann (von Neumann, 1932), they represent logical statements. A logic statement relates cognition, and, in particular, a semantic act.

Here is the central question that we outline. Our historical position in physical science as well as in psychology and neuroscience is to retain that matter is placed there in space and time existing as an objective entity, and independent from our human mental entities. These last entities operate in the abstract sphere and in condition of total separation and independence but also of constant interaction with external objective material reality. We are accustomed to consider reality as affected by a definite dualism. From one side we have matter that exists per se independently from our observation, measurement, cognition, etc. From the other side we have human cognitive structure and function. It is able to receive inputs from the outside sphere and to elaborate such information by performing semantic acts and decisions.

The previously written relation, $A=\sum_{i=1}^{k} a_{i} P_{i}$, indicates just the opposite. We have the observable $A$ that relates our material system (e.g. position of a particle, translational momentum, orbital angular momentum, spin, total angular momentum, energy, etc.), we have $a_{1}, a_{2}, \cdots, a_{k}$ that are the possible values of the dynamical variable that we are estimating in a probabilistic manner but, in addition, we have also $P_{1}, P_{2}, \cdots, P_{k}$ that represent logical statements and thus cognition. Looking again at the previous formula we deduce that quantum mechanics has a peculiar feature. It links at the same time material and cognitive entities. And this is a feature that in all the physics pertains only to quantum mechanics. By it we cannot reconcile the classical previously mentioned dualism matter-cognition or, generally speaking, matter-mind. We have a theory that instead blends together both of these aspects. Quantum mechanics and quantum cognition results point out that the traditional way that we walk considering materials and cognitive entities separated from each other is not acceptable. We retain that this is just the case. Ab initio we have primary cognitive entities coexisting with matter. The conclusion arising from our studies, based on quantum mechanics, seems to move in this direction. This is to say that, according to such results, quantum mechanics contains some basic features evidencing that its origin is also in the logic and in the cognition. It contains ab initio indication of our primary cognitive elements (Conte, 2011b, 2011c, 2013a, 2013b). In particular, the wave function of quantum mechanics, that is the pillar of the theory, must be considered as a factor of knowledge that engages our cognitive performance. 


\section{Conclusion}

The subsequent conclusion is that there are stages of our reality in which it results impossible to unconditionally defining the truth. Logic, language and thus cognition enter with a so fundamental role in quantum mechanics because there are levels of our reality in which the fundamental features of cognition and thus of logic and language, and thus the conceptual entities, acquire the same importance as the features of what is being described. At this level of reality we no more may separate the features of matter per se from the features of the cognition, of the logic, and of the language that we use to describe it. Conceptual entities non more are separated from the object of cognitive performance.

We retain that the current quantum cognition studies contribute to understand as cognition arose $a b$ initio and its present dynamics.

\section{References}

Aerts, D. (1991). A Mechanistic Classical Laboratory Situation Violating the Bell Inequalities with 2sqrt(2), Exactly in the Same Way as Its Violation by the EPR. Helvetica Physica Acta, 64, 1-23.

Busemeyer, J. R., \& Bruza, P. D. (2012). Quantum Models of Cognition and Decisions. Cambridge: Cambridge University Press. http://dx.doi.org/10.1017/CBO9780511997716

Conte, E. (2011a). Advances in Application of Quantum Mechanics in Neurosceinec and Psychology: A Clifford Algebraic Approach. New York: Nova Science Publishers.

Conte, E. (2011b). On the Logical Origins of Quantum Mechanics Demonstrated by Using Clifford Algebra. Neuroquantology, 9, 231-242. http://dx.doi.org/10.14704/nq.2011.9.2.397

Conte, E. (2011c). On the Logical Origins of Quantum Mechanics Demonstrated by Using Clifford Algebra: A Proof That Quantum Interference Arises in a Clifford Algebraic Formulation of Quantum Mechanics. Electronic Journal of Theoretical Physics, 8, 109-126.

Conte, E. (2013a). Are Information, Cognition, and the Principle of Existence Intrinsically Structured in the Quantum Model of Reality? Advanced Studies in Theoretical Physics, 7, 797-818.

Conte, E. (2013b). A Clifford Algebraic Analysis Gives Mathematical Explanation of Quantization of Quantum Theory and Delineates a Model of Quantum Reality in Which Information, Primitive Cognition Entities and a Principle of Existence Are Intrinsically Represented ab Initio. World Journal of Neuroscience, 3, 157-170. http://dx.doi.org/10.4236/wjns.2013.33021

Khrennikov, A. (2010). Ubiquitous Quantum Mechanics. Berlin Heidelberg: Springer-Verlag. http://dx.doi.org/10.1007/978-3-642-05101-2

Snyder, M. D. (1995). On the Quantum Mechanical Wave Function as a Link between Cognition and the Physical World: A Role for Psychology. cogprints.org/2196

Von Neumann, J. (1932). Mathematical Foundations of Quantum Mechanics. NY: Princeton University Press. 